\title{
Energy Cycle Optimization of Mobile Phones Using A Sleep Cycle Optimization Algorithm
}

\author{
Sadia Murawwat \\ Electrical Engineering Department \\ Lahore College for Women University \\ Lahore, Pakistan \\ Hira Akash \\ Electrical Engineering Department \\ Lahore College for Women University \\ Lahore, Pakistan
}

\author{
Iqra Manzoor \\ Electrical Engineering Department \\ Lahore College for Women University \\ Lahore, Pakistan \\ Javeria Ahmed \\ Electrical Engineering Department \\ Lahore College for Women University \\ Lahore, Pakistan
}

\begin{abstract}
Researchers and mobile network operators are dealing with the steep rise in energy consumption of mobile phones. A massive amount of phones are available in the market which are updating services from time to time. The problem lies in their battery drainage in response to the usage of internet-based applications including online streaming, multimedia requests, social networking usage and many more. In this research, a new technique is proposed and evaluated for energy optimization in mobile phones by exploiting the listening cycle of the mobile station (MS) wake-up mode while connected to the base station (BS) that is connected to a mobile network based on IEEE 802.16e. An MS cannot communicate with the BS if it is in the sleep mode while the other is in the wake-up mode. Service time and sojourn time are assumed to be exponential and based on tdistribution. The confidence interval comes up to $95 \%$ for our technique. The relative error is computed between the earlier techniques available and the approximated proposed one. Results show that there is a considerable improvement in energy consumption as compared to the other available techniques.
\end{abstract}

Keywords-energy optimization; mobile phones; listening interval; relative error

\section{INTRODUCTION}

The growth of Broadband Wireless Access (BWA) technologies is strongly related to the growing incorporation of internet and wireless multimedia applications of mobile phones in a wide variety of every day life's aspects. Worldwide Interoperability for Microwave Access (WIMAX) standards provide multiple physical layer (PHY) and Media Access Control (MAC) options and are a group of communication standards based on the IEEE 802.16 set of standards. The basic IEEE 802.16 was released in 2002 (originally focused on just fixed node stations) [1] and was modified later [2-4] in order to include mobility demands such as low energy consumption and techniques as the Sleep Mode Operation (SMO). Energy consumption and restoration has been the focusing point of several works [5-11]. The SMO technique uses two different modes for each Mobile Station (MS): the sleep mode and the wake-up mode. The MS falls in the sleep mode when it cannot transmit or receive uplink or downlink traffic to or from the Base Station (BS), thus saving power, while it switches to wake-up mode when it can transmit or receive from the BS.

SMO plays an essential role in the minimization of energy consumption of MS in an IEEE 802.16e based network. Sleep cycle and listening interval are the two main parts of sleep mode while the wake-up mode has respectively the active cycle and the listening interval. In SMO, sleep and wake-up mode can be switched from one to another in reference to the traffic arrival rate. When the MS is in wake-up mode and there is no uplink or downlink traffic for BS, it sends a Sleep Request (SLP_RQT) message addressed for BS to initiate sleep mode and waits for approval. If the BS responds positively, then the MS gets to sleep mode for a while and then wakes up temporarily for a small duration. This momentarily wake-up duration is called listening interval. If the BS responds negatively then the MS will remain in wake-up mode. Positive response indicates that there is no traffic and the MS can switch mode to wake-up. Negative response means that the MS should remain in the sleep mode. Sleep request and sleep response messages have some parameters i.e. initial and final sleep window, listening window and start interval of sleep mode. The minimum and maximum sleep intervals are represented as $T_{\min }$ and $T_{\max }$ respectively [12].

\section{THE SCOA ALGORITHM}

Sleep Cycle Optimization Algorithm (SCOA) is the here proposed algorithm for the minimization of energy usage in mobile stations using IEEE 802.16e. At first some parameters are initialized, i.e. $T_{\min }$ (sleep interval-minimum), $T_{\max }$ (sleep interval-maximum), $L$ (listening interval), where $E_{s}$ and $E_{L}$ represent the units of energy consumption per time in sleep cycles and listening cycles respectively. For the optimization of sleep cycle, minimum and maximum sleep and listening intervals are considered as key parameters. Sleep cycle is optimized by calculating the $j$ th sleep cycle using (1): 


$$
T_{j}=\left\{\begin{array}{l}
4^{j-1} T_{\min } \text { if } 4^{j-1} T_{\min }<T_{\max } \\
T_{\max } \text { if } 4^{j-1} T_{\min } \geq T_{\max }
\end{array}\right.
$$

In (1), $T_{j}$ represents the $j$ th sleep cycles and $j$ initializes from $j=1$. This calculation ensures that the sleep cycles are adjusted by increasing four times the time interval to the next sleep cycle as compared to the previous cycle instead of simply increment or doubling the previous sleep cycles. The algorithmic steps are:

1. Parameter initialization: $j=1, L=1 \mathrm{~ms}, \quad T_{\min }=1 \mathrm{~ms}$, $T_{\max }=1024 \mathrm{~ms}$

\section{Mode detection}

3. If wake-up mode, get $T_{\min }$, and $T_{\max }$

//Else (Step 4 )

Send sleep request message to $\mathrm{BS}$

MS will wait for response from BS

If MS gets positive response then it goes to step 4

If MS gets negative response then it goes to step 3

4. If sleep mode, compare values of $T_{\min }$, and $T_{\max }$

If $\left(T_{\min }<T_{\max }\right)$

Calculate $T_{j}$, using (1)

\section{$T_{j}: T_{\max }$}

Else

$T_{j}=T_{\max }$

5: Return to step 2

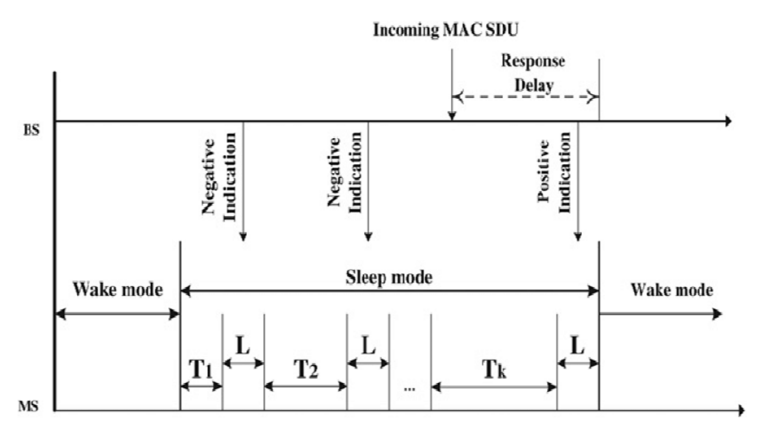

Fig. 1. Sleep mode operation

\section{NUMERICAL ANALYSIS}

The mathematical model of SCOA using SMO of IEEE $802.16 \mathrm{e}$ is presented in this section. The performance parameter i.e. energy consumption is evaluated by assuming that the frame arrival process follows the Poisson distribution with rate $\lambda$ and service time and sojourn time follow the same distribution with $1 / \lambda$. Frame arrival rates are recursively calculated for SCOA.

$$
\operatorname{Pr}\left(e_{j}=\text { True }\right)=1-\operatorname{Pr}\left(e_{j}=\text { False }\right)
$$

where,

$$
\operatorname{Pr}\left(e_{j}=\text { False }\right)=e^{-\lambda\left(T_{j}+L\right)}
$$

Equations (2)-(3) are the Poisson distribution equations of sleep mode parameters for data arrival and for no data arrival at MS respectively.

\section{ENERGY CONSUMPTION \& CONFIDENCE INTERVAL}

The energy consumption, $E[C]$ can be determined by adding the $E_{S}$ and $E_{L}$ to each sleep cycle time [13].The value of $E_{L}$ is $100 \mathrm{~mW}$ in listening interval of wake-up mode and $E_{S}$ is assumed to be $10 \mathrm{~mW}$. Hence, $E[C]$ is recursively calculated using (6):

$$
\begin{aligned}
& E[C]=\sum_{j=1}^{\infty} \operatorname{Pr}(n=j) \sum_{k=1}^{j}\left(T_{k} E_{S}+L E_{L}\right) \\
& E[C]=\sum_{J=1}^{\infty} e^{-\lambda \sum_{i=1}^{j-1}\left(T_{i}+L\right)}\left(1-e^{-\lambda\left(T_{j}+L\right)}\right) \sum_{k=1}^{j}\left(T_{k} E_{S}+L E_{L}\right) \\
& E[C]=\sum_{J=1}^{\infty} e^{-\lambda \sum_{i=1}^{j-1}\left(T_{i}+L\right)} \sum_{k=1}^{j}\left(T_{k} E_{S}+L E_{L}\right)- \\
& \sum_{J=1}^{\infty} e^{-\lambda \sum_{i=1}^{j}\left(T_{i}+L\right)} \sum_{k=1}^{j}\left(T_{k} E_{S}+L E_{L}\right)
\end{aligned}
$$

A $95 \%$ confidence interval of the proposed technique for the MS energy consumption has been calculated using (7), based on t-distribution that provides a predictable range of values with the assumption of $\alpha=0.05$. In (7), $\bar{x}$ represents mean, $s$ is the standard deviation, $n$ is the total sample number, and $t+(\alpha 2,(v))$ is calculated from the t-table.

$$
\bar{x} \pm t \alpha / 2,(v) \frac{s}{\sqrt{n}}
$$

\section{SIMULATION RESULTS \& COMPARISON}

The accuracy of the mathematical model is verified through MATAB simulations. The simulation results demonstrate the dependency relationships between the performance parameters and the frame arrival rate. The performance parameter, i.e. the energy consumption $E[C]$ was measured and compared for different values of $T_{\max }$. The arrival rate $(\lambda)$ was considered from 0.01 to 0.02 . Moreover, IEEE 802.16e standard and Advanced Energy Saving Mechanism (AESM) [5] approaches were compared with the proposed SCOA technique. The energy consumption comparison for the different techniques is summarized in Figure 2, where the sleep window-minimum is $1 \mathrm{msec}$ and the sleep window-maximum is $1024 \mathrm{msec}$. From Figure 2 it can be seen that IEEE 802.16e drains more energy per frame than SCOA due to its unnecessary energy consumption in sleep window because of the big number of sleep cycles. The comparison of the optimized value of energy consumption is shown in Table I. It is seen that SCOA consumes less energy than IEEE 802.16e and AESM.

\section{CONCLUSION}

In this research, Sleep Cycle Optimization Algorithm (SCOA) based on SMO has been compared with the basic IEEE 802.16e standard and the AESM approach with the use of MATLAB. The simulation results of the mathematical model of energy consumption have shown satisfactory results when compared to earlier existing techniques. Additionally, the confidence interval and relative error between the proposed technique and the basic standard IEEE 802.16e have been calculated. The confidence interval comes up to [1.99903, 1.99976] which shows efficient accuracy. The relative error 
between the standard and the proposed technique is 6.898 . Concluding, SCOA consumes $10 \%-20 \%$ less energy than the existing techniques.

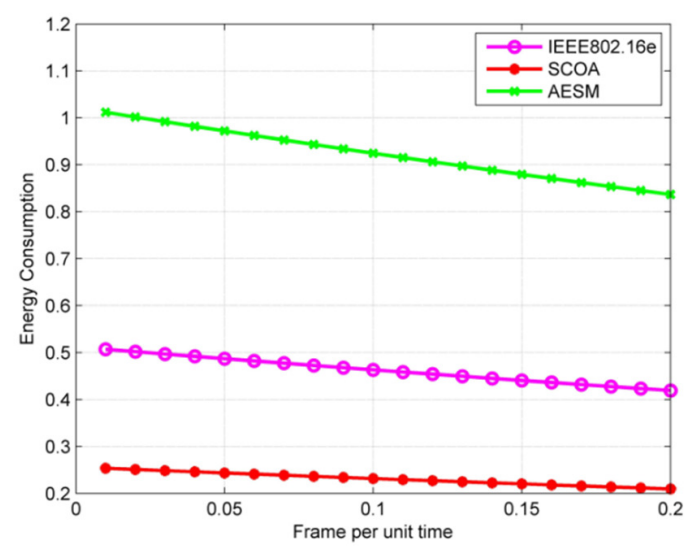

Fig. 2. Simulation of $E[C]$ for different techniques

TABLE I. ENERGY CONSUMPTION COMPARISON

\begin{tabular}{|c|c|c|c|}
\hline Frame per unit time $(\boldsymbol{\lambda})$ & IEEE802.16e & AESM & SCOA \\
\hline 0.02 & 2.004 & 4.004 & 0.2509 \\
\hline 0.04 & 1.965 & 3.925 & 0.2459 \\
\hline 0.06 & 1.926 & 3.847 & 0.241 \\
\hline 0.08 & 1.887 & 3.771 & 0.2362 \\
\hline 0.1 & 1.85 & 3.696 & 0.2315 \\
\hline 0.12 & 1.813 & 3.623 & 0.2269 \\
\hline 0.14 & 1.777 & 3.551 & 0.2224 \\
\hline 0.16 & 1.742 & 3.48 & 0.218 \\
\hline 0.18 & 1.707 & 3.411 & 0.2136 \\
\hline 0.2 & 1.673 & 3.344 & 0.2094 \\
\hline
\end{tabular}

\section{REFERENCES}

[1] IEEE, IEEE Std 802.16-2001-IEEE Standard for Local and Metropolitan Area Networks - Part 16: Air Interface for Fixed Broadband Wireless Access Systems, IEEE, 2002

[2] IEEE, IEEE Std 802.16e-2005-IEEE Standard for Local and Metropolitan Area Networks - Part 16: Air Interface for Fixed and Mobile Broadband Wireless Access Systems - Amendment for Physical and Medium Access Control Layers for Combined Fixed and Mobile Operation in Licensed Bands, IEEE, 2006

[3] IEEE, IEEE Std 802.16e/D6-2005-IEEE Amendment to IEEE Standard for Local and Metropolitan Area Networks - Part 16: Air Interface for Fixed Broadband Wireless Access Systems - Physical and Medium Access Control Layers for Combined Fixed and Mobile Operation in Licensed Bands, IEEE, 2005

[4] IEEE, IEEE Std 802.16e/D5-2004-IEEE, Amendment to IEEE Standard for Local and Metropolitan Area Networks - Part 16: Air Interface for Fixed Broadband Wireless Access Systems - Physical and Medium Access Control Layers for Combined Fixed and Mobile Operation in Licensed Bands, IEEE, 2004

[5] H. J. Thontadharya, D. Shwetha, M. S. Bhat, J. T. Devaraju, "Effect of Idle Mode on Power Saving in Mobile WiMAX Network", International Conference on Advances in Computing, Bangalore, India, July 4-6, 2012

[6] Y. Xiao, "Performance Analysis of an Energy Saving Mechanism in the IEEE 802.16e Wireless MAN", 3rd IEEE Consumer Communications and Networking Conference, Las Vegas, USA, January 8-10, 2006

[7] K. Han, S. Choi, "Performance Analysis of Sleep Mode Operation in IEEE 802.16e Mobile Broadband Wireless Access Systems", 63rd
Vehicular Technology Conference, Melbourne, Australia, May 7-10, 2006

[8] Y. Zhang, M. Fujise, "Energy management in the IEEE 802.16e MAC", IEEE Communications Letters, Vol. 10, No. 4, pp. 311-313, 2006

[9] J. B. Seo, S. Q. Lee, N. H. Park, H. W. Lee, C. H. Cho, "Performance Analysis of Sleep Mode Operation in IEEE 802.16e", 60th Vehicular Technology Conference, Los Angeles, USA, September 26-29, 2004

[10] N. H. Lee, S. Bahk, "MAC Sleep Mode Control Considering Downlink Traffic Pattern and Mobility",61st Vehicular Technology Conference, Stockholm, Sweden, May 30-June 1, 2005

[11] Y. Xiao, "Energy saving mechanism in the IEEE 802.16e wireless MAN", IEEE Communications Letters, Vol. 9, No. 7, pp. 595-597, 2005

[12] Y. Zhang, M. Fujise, "Energy management in the IEEE 802.16e MAC", IEEE Communications Letters, Vol. 10, No. 4, pp. 311-313, 2006

[13] Y. C. Tseng, J. J. Chen, Y. C. Yang, "Managing power saving classes in IEEE 802.16 wireless MANs: A fold-and- demultiplex method", Transactions on Mobile Computing, Vol. 10, No. 9, pp 1237-1247, 2011 